\title{
Site-specific endometrial injury improves implantation and pregnancy in patients with repeated implantation failures
}

\author{
Shang Yu Huang ${ }^{\dagger}$, Chin-Jung Wang ${ }^{\dagger}$, Yung-Kuei Soong, Hsin-Shih Wang, Mei Li Wang, Chieh Yu Lin and \\ Chia Lin Chang*
}

\begin{abstract}
Background: To test whether a site-specific hysteroscopic biopsy-induced injury in the endometrium during the controlled ovarian hyperstimulation cycle improves subsequent embryo implantation in patients with repeated implantation failure, a total of 30 patients who have had good responses to controlled ovulation stimulation but have failed to achieve pregnancy after two or more transfers of good-quality embryos were recruited in this prospective study.
\end{abstract}

Methods: A single, site-specific hysteroscopic biopsy-induced injury was generated on the posterior endometrium at midline 10-15 mm from the fundus during the D4-D7 period of the ongoing controlled ovarian hyperstimulation cycle in six patients.

Results: Patients received endometrial biopsy protocol achieved a pregnancy rate of 100\%. By contrast, only $46 \%$ of patients with similar clinical characteristics $(N=24)$ achieved pregnancy without the hysteroscopic biopsyinduced endometrium injury $(p<0.05)$.

Conclusions: Our proof-of-concept study demonstrates that a site-specific hysteroscopic endometrium injury performed during the ongoing in vitro fertilization (IVF) cycle, instead of injuries received during prior cycles, significantly improves clinical outcomes in patients with repeated implantation failure.

Keywords: hysteroscopy, endometrium biopsy, IVF, repeated implantation failure, pregnancy

\section{Background}

In assisted reproductive technology, procedures for culturing and transferring embryos have been continually improved over the last two decades. Yet the clinical pregnancy rate has not substantially improved over the last ten years (currently only $32.4 \sim 33.0 \%$ per IVF transfer as reported by ESHRE in 2010)[1], and many patients have suffered repeated implantation failure even in the most successful in vitro fertilization (IVF) clinics. Although no practical solutions for repeated implantation failure have emerged, an improved ability to control

\footnotetext{
* Correspondence: amego@cgmh.org.tw

+ Contributed equally

Department of Obstetrics and Gynecology, Chang Gung Memorial Hospital Linkou Medical Center, Chang Gung University, 5 Fu-Shin Street, Kweishan,

Taoyuan, Taiwan
}

C) Biomed Central

C 2011 Huang et al; licensee BioMed Central Ltd. This is an Open Access article distributed under the terms of the Creative Commons Attribution License (http://creativecommons.org/licenses/by/2.0), which permits unrestricted use, distribution, and reproduction in any medium, provided the original work is properly cited.

the endometrial environment for implantation promises to have a significant, positive impact on IVF outcomes.

Among the various potential causes of repeated implantation failure, uterine factors (e.g., thin endometrium, poor endometrial receptivity, and immunological incompatibility) have received the most attention in recent years [2]. It has been shown that endometrial receptivity could be modulated by a multitude of signaling molecules, including prostaglandins [3], growth factors, cytokines, chemokines, integrins, leukemia inhibitory factor [4,5], Wnt family ligands [6], and Ecadherin [7]. Whereas dysregulation of some of these factors could be associated with repeated implantation failure, key molecular mechanisms that underlie the regulation of endometrial receptivity remain to be elucidated [8]. 
Interestingly, earlier studies have shown that prior incidences of hysteroscopic endometrium biopsy are associated with increased rates of implantation, clinical pregnancy, and live birth among women who experienced repeated implantation failure but without obvious endometrium defects, suggesting that a hysteroscopic procedure in the nonconceptual cycle itself could be beneficial for improving pregnancy in subsequent IVF cycles [2,9-13]. This hypothesis has been supporteddirectly or indirectly-in a number of clinical settings [9-13]. Although earlier studies aiming to understand the effect of hysteroscopic endometrium biopsy on implantation have been methodologically diverse, a common denominator appears to have been the presence of local injuries prior to an IVF cycle. Because local inflammatory and angiogenesis reactions are indispensable for a successful implantation [14], an endometrial biopsy-induced injury could produce just such a local inflammatory and angiogenic environment between the endometrium and the conceptus, which, in turn, facilitates embryo implantation and subsequent pregnancy in earlier studies $[9,15,16]$.

However, due to substantial variations in patient selection, timing, number and extent of endometrial injury applied, and techniques in earlier studies [9,10,17-19], the merits of endometrial biopsy injury on clinical outcomes in IVF clinics remain controversial [2]. In the present study, we reasoned that, if a local injury is the key causal agent for improved pregnancy outcomes, then a single site-specific mechanical injury performed shortly before a scheduled embryo transfer should yield a similar result. Herein, we demonstrate that a standard IVF protocol in which a minimal intervention in the form of a site-specific endometrial injury (which was not intended for diagnostic or operative purposes) during the controlled ovarian hyperstimulation cycle leads to a dramatic improvement in the pregnancy rate of patients with repeated implantation failure.

\section{Methods}

\section{Subjects}

The study was approved by the institutional ethics committee review board of Chang Gung Memorial Hospital Linkou Medical Center. Institutional Review Board approval was obtained in accordance with the Helsinki Declaration of 1975 on human experimentation. All patients gave written informed consent to participate in the study. Repeated implantation failure was defined as the failure to conceive following at least two cycles of IVF and the transfer of good-quality embryos. Select patients who have consent to receive the hysteroscopic procedure during the ongoing hyperstimulation cycle were recruited for the site-directed hysteroscopic biopsy treatment.

\section{Hyperstimulation protocol and hysteroscopic procedure} Ovulation induction was performed by the administration of recombinant FSH (175 - 225 IU/day, Puregon, N.V. Organon, The Netherlands) starting on day 2 of the menstrual cycle. GnRH antagonist $(0.125 \mathrm{mg} /$ day, Cetrorelix, Serono) was given beginning on day 5 of rFSH injection, or when the leading follicle reached 10 $\mathrm{mm}$, to the day of hCG administration.

The single site-specific hysteroscopic biopsy procedure was performed using an electronic gynecological examination chair with the patient in a semirecumbent position. No premedication or local anesthetic was used. A Cuscoe's speculum was inserted into the vagina in order to visualize the cervix. Panoramic hysteroscopy was performed using a $4.9 \mathrm{~mm}$ diameter flexible hysteroscope (HYF-1T, Olympus Corporation, Shinjuku-ku, Tokyo, Japan). A 5\% Dextrose distending medium was propelled by an electronic pump (Endomat, Kar Storz, Tuttlingen, Germany) with an intrauterine pressure of approximately $45 \mathrm{~mm} \mathrm{Hg}$. A claw forceps (A4033, Olympus Corporation, Shinjuku-ku, Tokyo, Japan) was introduced through a $2.2 \mathrm{~mm}$ working channel, and used to generate a local injury on the posterior endometrium at midline $10-15 \mathrm{~mm}$ from the fundus on D4 to D7 of the stimulation cycle. The depth and width of the injured site was $2 \times 2 \mathrm{~mm}$ (i.e., a bite of the claw forceps). No antibiotic or hemostatic drug was administered after the procedure.

Once the leading follicle reached a size of at least 16 mm, 10, 000 IU of urinary hCG (Pregnyl 5, 000 IU, N.V. Organon, The Netherlands) was administrated to trigger ovulation. Ovum retrieval was performed $34 \mathrm{hr}$ after the hCG injection. Embryos were transferred transvaginally on day 3 of culture or at the blastocyst stage, and the tip of the catheter (Labotect Gmbh, Germany) was placed at $10-15 \mathrm{~mm}$ from the uterine fundus with a transfer volume of $\sim 10 \mu \mathrm{l}$. The luteal phase was supported with progesterone ( $8 \%$ vaginal-gel, Crinone, Prochieve). Clinical pregnancy was defined as visualization of fetal cardiac activity on transvaginal ultrasound examination.

\section{Statistical analysis}

Results were presented as mean \pm SD and analyzed by the Student's paired $t$-test. A $p$ value $<0.05$ was considered statistically significant.

\section{Results}

During a 6-month period (March to October 2010), a total of 30 patients with predominant diagnoses of tubal factor $(n=7)$ or unexplained infertility $(n=23)$ were recruited. From this group, 6 patients who had consent to the procedure were subsequently treated with the hysteroscopic biopsy protocol. Two of these patients 
had predominant diagnoses of tubal factor, and four had unexplained infertility. These participants ranged in age from 31 to 39 (mean = $34.0 \pm 3.0$ ), and the mean number of previous IVF cycle attempts for these cases was $2.8 \pm 0.8$. In these patients, there was no difference in total dose of gonadotropin used, $E_{2}$ levels on the day of hCG administration, fertilization rate, and the number of embryos transferred between previous unsuccessful cycles and the cycle during which the hysteroscopic biopsy-induced endometrium injury was performed (Figure 1, Table 1). In these patients, there was a trend toward having a thicker endometrium in cycles undergoing endometrial biopsy; however, the difference did not reach statistical significance $(10.3 \pm 2.0 \mathrm{~mm}$ vs. 8.7 $\pm 2.2 \mathrm{~mm}, p=0.26$ ). In addition, we observed a thin hypoechogenic endometrium on the day of endometrial biopsy in all patients, and a triple-line multi-layered pattern of endometrium was found on the day of hCG administration. The hysteroscopic technique obviously did not alter this expected transition in endometrial lining pattern.

Of importance, we found that the implantation rate of patients in the treatment group was 56\% (Tables 1 and 2 ), and a total of six pregnancies (100\%)-four singleton, one twin, and one triplet-were recorded after the hysteroscopic intervention cycles. By contrast, 24 patients who had similar clinical characteristics but did not receive the hysteroscopic intervention recorded only a $21 \%$ rate of implantation per transferred embryo and a

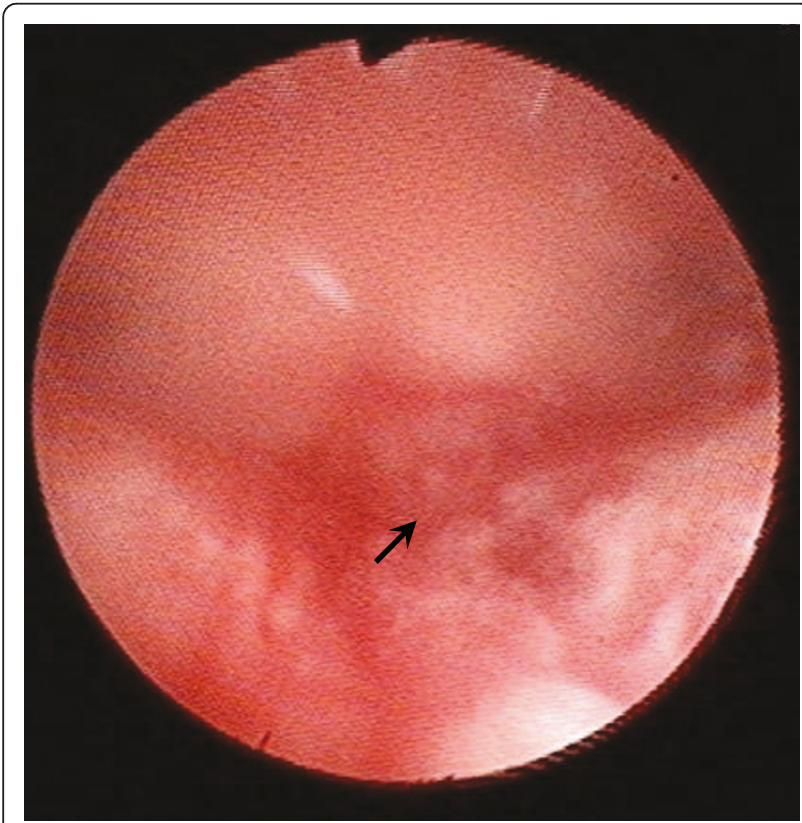

Figure 1 Image of an operation during which an injury in the endometrium was generated using a flexible hysterofibrescope. The injured site is indicated by an arrow.
$46 \%(11 / 24)$ clinical pregnancy rate (Table 2$)$. Consistently, the control group has a higher early abortion rate $(27 \%(3 / 11))$ as compared to the treatment group $(0 \%$ $(0 / 6))$ at the $12^{\text {th }}$ week of gestation (Additional file 1 , Supplemental Table S1).

\section{Discussion}

Although major advances have been made in improving oocyte and embryo development in IVF clinics in the last two decades, hurdles remain in achieving high embryo implantation rates for many patients. For both patients and clinicians, one of the most discouraging issues in IVF treatments is the recognition of repeated implantation failure after transfers of good-quality embryos because it has been generally accepted that pregnancy and implantation rates are significantly lower for patients who undergo their second or third cycles of treatment as compared to those undergoing their first cycle of IVF [20].

Whereas causes of defective implantation in patients with repeated implantation failure could be multi-factorial or even originate from embryonic defects (e.g., genetic abnormalities and embryonic aneuploidy), earlier observations noted that patients who had received hysteroscopic biopsies prior to subsequent IVF cycle for various reasons had a higher pregnancy rate [2]. This observation has led to the hypothesis that endometrium injury might improve pregnancy in patients with repeated implantation failure as a result of subsequent inflammatory responses and changes in cytokine production in the endometrium [9-11,21]. Although a number of subsequent studies have reported significant correlations between incidence of hysteroscopic biopsy and improved pregnancy; however, it has not been directly defined what injury per se is the causal agent for the observed improvement because almost all earlier studies have applied blind and nonspecific injuries during cycles prior to the final controlled ovarian hyperstimulation cycle. In addition to variations in the type and extent of injuries received by patients, it is conceivable that the effect of endometrium injuries given long before the subsequent embryo-transferring cycle could be lessened by subsequent menses.

Because the exact injury and timing required to effectively improve implantation is not clear, the value of hysteroscopic injury for improving pregnancy in patients with repeated implantation failure remains to be established. To specifically investigate whether a local injury itself is sufficient to improve implantation, we applied a hysteroscopic biopsy at a specific site of the endometrium (posterior wall 10-15 $\mathrm{mm}$ from the fundus) during a specific time window (D4 to D7) of the ongoing stimulation cycle-instead of relying on nonspecific injuries from prior cycles. This design has effectively eliminated 
Table 1 Clinical characteristics of patients who received a site-specific endometrium injury during the final IVF cycle

\begin{tabular}{|c|c|c|c|}
\hline Characteristics & The IVF cycle with a biopsy & Previous failed cycles ${ }^{a}$ & $p$ value $^{\mathrm{b}}$ \\
\hline Length of stimulation (day) & $8.7 \pm 1.0$ & $9.9 \pm 1.3$ & 0.11 \\
\hline Total units of gonadotropin administered & $1570 \pm 377$ & $1893 \pm 270$ & 0.10 \\
\hline Endometrium thickness on the day of hCG administration ( $\mathrm{mm}$ ) & $10.3 \pm 2.0$ & $8.7 \pm 2.2$ & 0.26 \\
\hline E2 levels on the day of $\mathrm{hCG}$ administration $(\mathrm{pg} / \mathrm{mL})$ & $1623 \pm 550$ & $1825 \pm 879$ & 0.64 \\
\hline Number of oocytes retrieved & $11.3 \pm 2.9$ & $7.1 \pm 3.1$ & 0.03 \\
\hline Fertilization rate (\%) & $59 \pm 29$ & $49 \pm 18$ & 0.52 \\
\hline Number of embryos transferred & $2.7 \pm 1.2$ & $2.3 \pm 1.1$ & 0.91 \\
\hline Implantation rate (sac number/transferred embryo number) & $56 \%$ & 0 & \\
\hline Pregnancy rate (clinical pregnancy/per ET) & $100 \%$ & 0 & \\
\hline
\end{tabular}

${ }^{a}$ Measurements are the mean of parameters recorded in previous failed cycles

${ }^{b}$ Student's $t$-test of comparisons between the ongoing and prior IVF cycles

many confounding effects associated with the extent and duration of nonspecific injuries administered in earlier studies. In addition, by incorporating the intervention process into the traditional IVF procedure, this study has allowed us to compare differences not only between treatment groups but also between the biopsy cycle and earlier failed cycles in these patients. Furthermore, we chose to perform the biopsy at a specific location of the endometrium because it has been recently shown that the deposition of embryos at 10 to $20 \mathrm{~mm}$ from the uterine fundus allows patients receiving IVF to reach a higher pregnancy rate [22]. Consistent with our hypothesis, the results demonstrated that a site-specific hysteroscopic biopsy-induced endometrium injury during the follicular phase of an ongoing IVF cycle can significantly improve implantation and pregnancy rates. This result is important because this hysteroscopic intervention protocol could become a standard procedure for treating repeated implantation failure. Nonetheless, it is important to note that our proof-of-concept study was performed with a small group of patients, and that there are differences in the total units of gonadotropin administered and the total number of previously transferred embryos between the two study groups (Table 2). Future studies with larger populations of patients are needed to validate current findings.

Table 2 Comparison of clinical characteristics and treatment outcomes of patients with or without a site-specific hysteroscopic biopsy-induced endometrium injury during the final IVF cycle

\begin{tabular}{|c|c|c|c|}
\hline Characteristics & $\begin{array}{l}\text { Patients with a site-specific endometrium injury } \\
\qquad(\mathrm{N}=6)\end{array}$ & $\begin{array}{l}\text { Control patients }(\mathrm{N}= \\
\text { 24) }\end{array}$ & $\begin{array}{c}p \\
\text { value }^{\mathrm{a}}\end{array}$ \\
\hline Mean age (yr) & $34 \pm 3.0$ & $35 \pm 4.1$ & 0.58 \\
\hline Weight (kg) & $53.4 \pm 3.0$ & $54.3 \pm 7.4$ & 0.80 \\
\hline $\mathrm{FSH}(\mathrm{mlU} / \mathrm{mL})$ & $6.7 \pm 2.3$ & $6.9 \pm 2.8$ & 0.86 \\
\hline $\mathrm{LH}(\mathrm{mlU} / \mathrm{mL})$ & $4.2 \pm 1.9$ & $4.3 \pm 2.1$ & 0.89 \\
\hline Duration of Infertility (yr) & $5.7 \pm 2.8$ & $6.4 \pm 2.9$ & 0.58 \\
\hline Total number of previously transferred embryos & $5.0 \pm 2.2$ & $7.8 \pm 3.0$ & 0.06 \\
\hline Total number of previously IVF/ICSI cycles & $2.8 \pm 0.8$ & $2.8 \pm 0.9$ & 0.88 \\
\hline Length of stimulation (day) & $8.7 \pm 1.0$ & $9.2 \pm 1.1$ & 0.24 \\
\hline Total units of gonadotropin administrated (IU) & $1570 \pm 377$ & $2206 \pm 513$ & 0.01 \\
\hline $\begin{array}{l}\text { Endometrium thickness on the day of hCG administration } \\
(\mathrm{mm})\end{array}$ & $10.3 \pm 2.0$ & $9.8 \pm 2.1$ & 0.62 \\
\hline E2 levels on the day of $\mathrm{hCG}$ administration $(\mathrm{pg} / \mathrm{mL})$ & $1623 \pm 550$ & $1822 \pm 1078$ & 0.67 \\
\hline Number of oocytes retrieved & $11.3 \pm 2.9$ & $13.5 \pm 7.4$ & 0.50 \\
\hline Number of fertilizations & $7.2 \pm 4.6$ & $6.3 \pm 4.0$ & 0.66 \\
\hline Fertilization rate (\%) & $59 \pm 29$ & $50 \pm 22$ & 0.45 \\
\hline Number of all embryos & $3.8 \pm 2.3$ & $4.8 \pm 2.0$ & 0.32 \\
\hline Number of embryos transferred & $2.7 \pm 1.2$ & $3.0 \pm 0.5$ & 0.29 \\
\hline Implantation rate (sac number/transferred embryo) & $56 \%$ & $21 \%$ & 0.01 \\
\hline Pregnancy rate (clinical pregnancy/per ET) & $100 \%$ & $46 \%$ & 0.04 \\
\hline $\begin{array}{l}\text { Early abortion rate (abortion before } 12 \text { wks/total } \\
\text { pregnancy) }\end{array}$ & $0 \%(0 / 6)$ & $27 \%(3 / 11)$ & \\
\hline
\end{tabular}

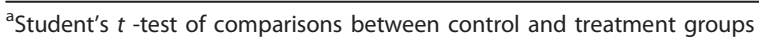


For a successful pregnancy in placental mammals, two major physiological events have to occur: (a) building a receptive uterine endometrium for embryo implantation, and (b) protecting the semiallotypic fetus from attacks by the maternal immune system. Although the exact mechanism by which the local injury improves pregnancy remains to be investigated, our study has strengthened the hypothesis that a mechanical injury may enhance uterine receptivity. By provoking the immune system with an injury, the immune response and inflammatory reactions associated with wound healing in the endometrium may, in turn, increase the endometrial receptivity to the semiallotypic embryo [23]. Alternatively, the local injury could generate a focus for the accumulation of uterine dendritic cells and accompanying increases in innate immune molecules [24], or provide an enhanced angiogenic environment enriched with cytokines and growth factors known to be essential for normal trophoblast invasion.

\section{Conclusions}

As a whole, our pilot study has provided direct evidence to support the hypothesis that a local injury performed during an ongoing IVF cycle is beneficial for implantation in patients with repeated implantation failure, and a discrete procedure for further testing the hypothesis that a site-specific mechanical injury could improve pregnancy outcomes. In addition, we envision that the establishment of this discrete procedure could provide a platform for systematic investigations of molecular mechanisms underlying endometrium injury-mediated improvement in implantation and the role of various inflammatory factors in regulating endometrium receptivity in humans.

\section{Additional material}

Additional file 1: Supplemental Table S1. Clinical outcomes of patients with a clinical pregnancy.

\section{List of abbreviations}

IVF: in vitro fertilization; FSH: follicle stimulating hormone; hCG: human chorionic gonadotropin.

\section{Acknowledgements}

We thank director Chyi-Long Lee, M.D. Ph.D., and chairperson Hong-Yuan Huang, M.D. at Chang Gung Memorial Hospital for their encouragement. We also thank Ms. Mei-Wen Lee for her technical assistance. No funding was sought or received to support the undertaking of this project. CLC is grateful to the support of Chang Gung Memorial Hospital.

\section{Authors' contributions}

CLC contributed to conception, design, and initiation of the study. CJW, MLW, CYL, and CLC performed the clinical procedures. SYH made substantial contributions to the analysis of samples and data. CLC, YKS, and HSW contributed to the interpretation of the data and the preparation of the manuscript. All authors read and approved the final manuscript.

\section{Competing interests}

The authors declare that they have no competing interests.

Received: 3 June 2011 Accepted: 21 October 2011

Published: 21 October 2011

\section{References}

1. de Mouzon J, Goossens V, Bhattacharya S, Castilla JA, Ferraretti AP, Korsak V, Kupka M, Nygren KG, Nyboe Andersen A: Assisted reproductive technology in Europe, 2006: results generated from European registers by ESHRE. Hum Reprod 2010, 25:1851-1862.

2. Margalioth EJ, Ben-Chetrit A, Gal M, Eldar-Geva T: Investigation and treatment of repeated implantation failure following IVF-ET. Hum Reprod 2006, 21:3036-3043.

3. Achache H, Tsafrir A, Prus D, Reich R, Revel A: Defective endometrial prostaglandin synthesis identified in patients with repeated implantation failure undergoing in vitro fertilization. Fertil Steril 2010, 94:1271-1278.

4. Aghajanova L: Leukemia inhibitory factor and human embryo implantation. Ann N Y Acad Sci 2004, 1034:176-183.

5. Kao LC, Tulac S, Lobo S, Imani B, Yang JP, Germeyer A, Osteen K, Taylor RN, Lessey BA, Giudice LC: Global gene profiling in human endometrium during the window of implantation. Endocrinology 2002, 143:2119-2138.

6. Tulac S, Nayak NR, Kao LC, Van Waes M, Huang J, Lobo S, Germeyer A, Lessey BA, Taylor RN, Suchanek E, Giudice LC: Identification, characterization, and regulation of the canonical Wnt signaling pathway in human endometrium. J Clin Endocrinol Metab 2003, 88:3860-3866.

7. Rahnama F, Thompson B, Steiner M, Shafiei F, Lobie PE, Mitchell MD: Epigenetic regulation of $\mathrm{E}$-cadherin controls endometrial receptivity. Endocrinology 2009, 150:1466-1472.

8. Horcajadas JA, Pellicer A, Simon C: Wide genomic analysis of human endometrial receptivity: new times, new opportunities. Hum Reprod Update 2007, 13:77-86.

9. Almog B, Shalom-Paz E, Dufort D, Tulandi T: Promoting implantation by local injury to the endometrium. Fertil Steril 2010, 94:2026-2029.

10. Bosteels J, Weyers S, Puttemans P, Panayotidis C, Van Herendael B, Gomel V, Mol BW, Mathieu C, D'Hooghe T: The effectiveness of hysteroscopy in improving pregnancy rates in subfertile women without other gynaecological symptoms: a systematic review. Hum Reprod Update 2010, 16:1-11.

11. Demirol A, Gurgan T: Effect of treatment of intrauterine pathologies with office hysteroscopy in patients with recurrent IVF failure. Reprod Biomed Online 2004, 8:590-594.

12. Makrakis $E$, Pantos $K$ : The outcomes of hysteroscopy in women with implantation failures after in-vitro fertilization: findings and effect on subsequent pregnancy rates. Curr Opin Obstet Gynecol 2010, 22:339-343.

13. Bozdag G, Aksan G, Esinler I, Yarali $H$ : What is the role of office hysteroscopy in women with failed IVF cycles? Reprod Biomed Online 2008, 17:410-415.

14. Quenby S, Nik H, Innes B, Lash G, Turner M, Drury J, Bulmer J: Uterine natural killer cells and angiogenesis in recurrent reproductive failure. Hum Reprod 2009, 24:45-54.

15. Gnainsky Y, Granot I, Aldo PB, Barash A, Or Y, Schechtman E, Mor G, Dekel $\mathrm{N}$ : Local injury of the endometrium induces an inflammatory response that promotes successful implantation. Fertil Steril 2010, 94:2030-2036.

16. Zhou L, Li R, Wang R, Huang HX, Zhong K: Local injury to the endometrium in controlled ovarian hyperstimulation cycles improves implantation rates. Fertil Steril 2008, 89:1166-1176.

17. Gavino-Gavino F, Guzman-Gonzalez E, Reyes-Munoz E, Villalpando-Bravo Jde J, Jauregui-Melendez RA: IImpact of office hysteroscopy in patients with a history of two or more failed cycles of IVF-ET and pre-ICSI in assisted an reproduction center]. Ginecol Obstet Mex 2010, 78:9-14.

18. Lorusso F, Ceci O, Bettocchi S, Lamanna G, Costantino A, Serrati G, Depalo R: Office hysteroscopy in an in vitro fertilization program. Gynecol Endocrinol 2008, 24:465-469.

19. Rama Raju GA, Shashi Kumari G, Krishna KM, Prakash GJ, Madan K: Assessment of uterine cavity by hysteroscopy in assisted reproduction 
programme and its influence on pregnancy outcome. Arch Gynecol Obstet 2006, 274:160-164.

20. Shapiro BS, Richter KS, Harris DC, Daneshmand ST: Dramatic declines in implantation and pregnancy rates in patients who undergo repeated cycles of in vitro fertilization with blastocyst transfer after one or more failed attempts. Fertil Steril 2001, 76:538-542.

21. Orvieto R, Meltcer S, Liberty G, Rabinson J, Anteby EY, Nahum R: A combined approach to patients with repeated IVF failures. Fertil Steril 2010, 94:2462-2464

22. Mains L, Van Voorhis BJ: Optimizing the technique of embryo transfer. Fertil Steril 2010, 94:785-790.

23. Garlanda C, Maina V, Martinez de la Torre Y, Nebuloni M, Locati M: Inflammatory reaction and implantation: the new entries PTX3 and D6. Placenta 2008, 29(Suppl B):129-134.

24. Dekel N, Gnainsky Y, Granot I, Mor G: Inflammation and implantation. Am J Reprod Immunol 2010, 63:17-21.

doi:10.1186/1477-7827-9-140

Cite this article as: Huang et al: Site-specific endometrial injury improves implantation and pregnancy in patients with repeated implantation failures. Reproductive Biology and Endocrinology 2011 9:140.

\section{Submit your next manuscript to BioMed Central} and take full advantage of:

- Convenient online submission

- Thorough peer review

- No space constraints or color figure charges

- Immediate publication on acceptance

- Inclusion in PubMed, CAS, Scopus and Google Scholar

- Research which is freely available for redistribution

Submit your manuscript at www.biomedcentral.com/submit 